\title{
Usefulness of serum lactate as a predictor of successful discontinuation of continuous atropine infusion in patients with severe acute organophosphate poisoning
}

\author{
Ho Chul Kwon, Yong Sung Cha, Gyo Jin An, Yoonsuk Lee, Hyun Kim \\ Department of Emergency Medicine, Yonsei University Wonju College of Medicine, Wonju, Korea
}

Objective In severe organophosphate (OP) poisoning, administration of atropine via continuous intravenous infusion is typically considered. To date, there have been no studies on predicting successful atropine discontinuation through plasma cholinesterase (PChE) and serum lactate levels, which are monitored during critical care in severe acute OP poisoning. Therefore, we retrospectively evaluated the usefulness of serum lactate and PChE as predictors of successful discontinuation of atropine infusion.

Methods This retrospective observational study was performed on consecutive adult patients treated for severe acute OP poisoning between March 2011 and December 2016. We sequentially evaluated serum lactate and PChE levels on emergency department arrival and before a discontinuation trial of atropine infusion. Discontinuation of atropine intravenous infusion was attempted in patients after clearance of respiratory secretions and cessation of bronchoconstriction. Discontinuation of atropine infusion attempts were divided into successful and failed trials.

Results A total of 95 trials were conducted in 62 patients. Serum lactate levels before trials were significantly different between patients with successful and failed trials. The area under the curve for prediction of successful atropine discontinuation using serum lactate levels before trial discontinuation were 0.742 (95\% confidence interval, 0.638 to 0.846$)$. PChE level was not significantly different between two groups.

Conclusion Serum lactate levels before the discontinuation trial of atropine infusion served to predict successful discontinuation in severe acute OP poisoning.

Keywords Organophsophate; Atropine; Prognosis

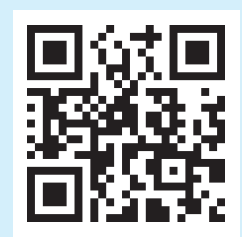

How to cite this article:

Kwon HC, Cha YS, An GJ, Lee Y, Kim H. Usefulness of serum lactate as a predictor of successful discontinuation of continuous atropine infusion in patients with severe acute organophosphate poisoning. Clin Exp Emerg Med 2018;5(3):177-184.
This is an Open Access article distributed under the terms of the Creative Commons Attribution Non-Commercial License (http:// creativecommons.org/licenses/by-nc/4.0/). 


Wapsule
What is already known
Summary
pine via continuous intravenous infusion is considered. The intravenous atropine infusion is then usually titrated after
checking the end-points of atropine therapy: clearance of respiratory secretions and cessation of bronchoconstriction.
However, even when the intravenous atropine infusion is succesffully titrated and then discontinued with satisfying
therapeutic end-points, cholinergic symptoms and signs often recur.
What is new in the current study
Serum lactate levels before a discontinuation trial of atropine infusion served as a predictor of successful discontinua-
tion trials in organophosphate poisoning.

\section{INTRODUCTION}

Because organophosphate (OP) insecticides irreversibly inhibit acetylcholinesterase (AChE), they cause accumulation of acetylcholine and overstimulation of cholinergic synapses in the central nervous system, somatic nerves, parasympathetic nerve endings, and sweat glands. 'The clinical features of acute cholinergic overstimulation include bronchorrhea, bronchospasm, bradycardia, lacrimation, salivation, miosis, urination, emesis, and diarrhea. ${ }^{2}$ Because of respiratory failure due to bronchorrhea and respiratory muscle weakness in OP poisoning, use of an antidote such as pralidoxime or atropine is very important. Atropine competes with acetylcholine at muscarinic receptors and prevents cholinergic activation., ${ }^{3,4}$ Severe OP poisoning requires large doses of atropine; after early atropinization, administration of atropine via continuous intravenous (IV) infusion is typically considered. Then, we usually titrate IV atropine infusion after checking the end-points for atropine therapy: clearance of respiratory secretions and cessation of bronchoconstriction. ${ }^{5}$ However, even when the IV atropine infusion is successfully titrated and then discontinued with satisfying therapeutic end-points, cholinergic symptoms and signs often recur. Repeated failure to discontinue atropine infusion may lead to increased morbidity and mortality related to atropine toxicity, and intensive care unit (ICU) admission may be prolonged. Therefore, it would be helpful for clinicians if a laboratory test could predict successful discontinuation of atropine infusion.

Measurement of serum lactate levels is important in assessing severely ill patients. Elevated blood lactate is associated with mortality among critically ill patients with and without infections. ${ }^{6,7}$ In critically ill OP-poisoned patients such as those with sepsis, we have used serial serum lactate for guidance in providing intensive care. ${ }^{8,9}$ Both clinical symptoms and laboratory markers such as AChE have been used to diagnose OP. Direct measurement of red blood cell AChE (R-AChE) activity provides a range for the degree of OP toxicity, and sequential measurement of R-AChE activity can also be used to determine the effectiveness of pralidoxime therapy in enzyme regeneration, but most hospital laboratories do not readily perform this test. ${ }^{10}$ Although plasma cholinesterase (PChE) does not correlate well with the severity of poisoning, measuring PChE levels is relatively easy. ${ }^{10}$

Serum lactate and PChE may be useful in guiding successful discontinuation of atropine infusion because association of successful discontinuation of atropine with high lactate and low PChE levels has been sometimes noted in our practice. However, to the best of our knowledge, no previous studies have investigated the clinical usefulness of serum lactate or PChE for predicting successful discontinuation of atropine infusion in patients with severe OP poisoning. Because our facility uses PChE and serum lactate levels for diagnosis and treatment of severe OP poisoning, PChE and serum lactate are measured sequentially during hospitalization. Therefore, we retrospectively evaluated the usefulness of serum lactate and PChE as predictors of successful discontinuation of atropine infusion.

\section{METHODS}

\section{Study setting and population}

This was a retrospective and observational study of consecutive adult patients diagnosed with acute OP insecticide poisoning who presented to the emergency department (ED) between March 2011 and December 2016. The ED in this study is located in a suburban tertiary-care hospital, has more than 44,000 annual visits, and is staffed 24 hours per day by board-certified emergency physicians.

OP poisoning was confirmed by patient or guardian statements, and verification of the agent was determined by an emergency physician who transcribed the bottle label into the patient records. Clinical manifestations consistent with OP poisoning and PChE levels were also used to confirm OP poisoning. OP-poisoned 
patients were treated with decontamination and administration of pralidoxime and atropine. The protocol for pralidoxime administration at our hospital is to infuse 1 to $2 \mathrm{~g}$ over 20 to $30 \mathrm{~min}$ utes followed by 0.5 to $1.0 \mathrm{~g} / \mathrm{hr}$ for at least 48 hours according to the severity of clinical conditions and the time course of PChE activity. For atropine, an initial IV bolus of 1 to $10 \mathrm{mg}$ depending on the severity of symptoms and continuous infusion was administered to patients with more than moderate poisoning according to the Namba classification."

The study exclusion criteria were (1) < 19 years old, (2) nonoral poisoning, (3) non-use of continuous atropine infusion owing to mild poisoning, (4) poisoning by any additional agent (with the exception of alcohol), (5) cardiac arrest on ED arrival, (6) transfer to another hospital after ED admission, (7) additional medical conditions such as chronic liver disease or malignant tumors, which can cause changes in PChE (PChE is primarily synthesized in the liver), and (8) insufficient data including unknown subtype of OP. Since the study was performed retrospectively and observationally, we did not obtain informed consent from participants. Approval of this study was obtained from the institutional review board (CR316122).

\section{Data collection}

Data were collected by retrospectively reviewing electronic medical records. Data collection was conducted by 2 emergency physicians who were blinded to the study objectives and hypothesis. Data abstractors were trained before data collection to reduce possible bias during data collection. We used explicit case report forms, and chart abstractors and study coordinators met periodically to resolve any disputes and to review coding rules. The study coordinators monitored the performance of the abstractors. Because the study was performed retrospectively and observationally, patient records and/or information were anonymized before analysis.

We collected demographic data (including age and sex), type of $O P$, Namba classification, ${ }_{1}^{11}$ ingested amount of $\mathrm{OP}$, elapsed time from ingestion to arrival at the $E D$, use of charcoal and gastric lavage, cause of poisoning, information regarding preexisting comorbidities, vital signs (systolic blood pressure, diastolic blood pressure, pulse rate, respiratory rate, and body temperature), initial mental status (Glasgow Coma Scale), and ST changes on an electrocardiogram. We investigated the use of vasoactive drugs such as norepinephrine or dopamine and mechanical ventilation as well as the presence of pneumonia during the trial discontinuation of atropine infusion. We evaluated complications, including pneumonia, endotracheal intubation, respiratory failure, decreased mental status, acute kidney injury, ${ }^{12}$ and hypotension during hos- pitalization. Total admission and ICU admission days and in-hospital mortality were also evaluated.

We estimated the amount of OP ingested as follows: "a little" or "a spoonful" was taken to be $5 \mathrm{~mL}$, "a mouthful" was presumed to be $25 \mathrm{~mL}$, "a small cup" was presumed to be $100 \mathrm{~mL}$, and "a bottle" was presumed to be $300 \mathrm{~mL}^{13}$ The chemical structure of $\mathrm{OP}$ is an important parameter in the rate of aging of human $\mathrm{AChE}_{i}$ the half-life of the aging process of AChE with dimethyl OP is 3.7 hours, whereas that with diethyl OP is 33 hours. ${ }^{14,15}$ Therefore, we divided the subjects into 2 groups according to the chemical formulation of OP ingested: the dimethyl subtype and the diethyl subtype. ${ }^{16}$ The unclassified subtype included subjects in which the ingested OP did not match either the dimethyl or diethyl classification. The severity of poisoning was classified as latent, mild, moderate, or severe using the Namba classification."

We evaluated arterial blood gas, serum lactate, and PChE levels on ED arrival (initial serum lactate or PChE) and within 12 hours before discontinuation of atropine infusion (serum lactate or PChE before trial discontinuation). If multiple measures of arterial blood gas, serum lactate, and PChE were taken within 12 hours before discontinuation of atropine infusion, the most recent value before the trial was selected. PChE levels (reference range, 7,000 to 19,000 $\mathrm{U} / \mathrm{L}$ ) were obtained using Dimension Vista (Siemens Healthcare Diagnostics, Newark, DE, USA).

At our facility, indications for atropine IV infusion discontinuation are clearance of respiratory secretions and cessation of bronchoconstriction. ${ }^{5}$ Atropine infusion discontinuation attempts were divided according to recurrence of cholinergic symptoms and signs. A successful trial was defined as no recurrence of cholinergic symptoms after discontinuing atropine infusion. A failed trial was defined as the need to restart the atropine infusion due to recurrence of cholinergic symptoms including bronchorrhea or bronchospasm.

\section{Study endpoint}

The primary outcomes of this study were the investigation of differences between successful and failed trials in terms of serum lactate and PChE levels checked within 12 hours before discontinuation of atropine infusion and investigation of the predictive value of serum lactate and PChE levels for successful discontinuation of atropine infusion in patients with severe OP poisoning.

\section{Statistical analysis}

Categorical variables are presented as frequencies and percentages, and continuous variables are presented as means and standard deviations or as medians and interquartile ranges. Normality was assessed using the Shapiro-Wilk test. The chi-square test or 
Fisher exact test was used to compare categorical variables, while the 2-sample t-test or the Mann-Whitney U-test was used to compare continuous variables. Variables with P-values of $<0.05$ by univariate analysis were entered into the multiple logistic regression analysis to factors predicting successful discontinuation of atropine infusion in patients with severe OP poisoning, and the results are expressed as odds ratio with 95\% confidence interval (CI). The area under the curve (AUC) for the predictive ability of variables was determined using receiver operating characteristic curves. P-values of $<0.05$ were considered statistically significant, and analyses were performed using IBM SPSS Statistics ver. 23 (IBM Corp., Armonk, NY, USA) and MedCalc Statistical Software ver. 17.5.3 (MedCalc Software, Ostend, Belgium).

\section{RESULTS}

\section{Patient characteristics}

A total of 87 consecutive adult patients with acute OP poisoning were identified during the study period. Twenty-five patients were excluded based on the following criteria: (1) < 19 years old (1 patient); (2) non-oral poisoning (1 patient); (3) non-use of continuous atropine infusion due to mild poisoning (2 patients); (4) poisoning by any additional agent (with the exception of alcohol) (8 patients); (5) cardiac arrest on ED arrival (4 patients); (6) transfer to another hospital after ED admission (1 patient); (7) additional conditions such as chronic liver disease or malignant tumors (2 patients); and (8) insufficient data including unknown subtype (6 patients). After exclusion, 62 patients with severe acute OP poisoning were enrolled in this study (Fig. 1).

A total of 95 atropine discontinuation attempts were made in 62 patients. Baseline characteristics and laboratory findings are shown in Table 1. Of the 62 analyzed patients, 47 (75.8\%) were male. The mean age was 60 years (range, 18 to 91 years). The dimethyl subtype was the most common type of OP poisoning (30 patients, 48.8\%). The dimethyl subtype included dichlorvos (10 patients), phosphamidon (14 patients), methidathion (4 patients), and fenthion (2 patients). The diethyl subtype included chlorpyrifos (13 patients), parathion (1 patient), and diazinon (2 patients). The median ingested amount was $100 \mathrm{~mL}$ and intentionality was present in 52 patients (83.9\%). The median initial Glasgow Coma Scale was 8, and initial PChE and serum lactate levels were 322 $\mathrm{U} / \mathrm{L}$ and $4.38 \mathrm{mmol} / \mathrm{L}$, respectively. Endotracheal intubation due to respiratory failure or unprotected airway due to decreased mental status (53 patients, 85.5\%) were the most common complications during hospitalization. Pneumonia and hypotension were found in 23 patients (37.1\%) and 19 patients (30.6\%), respectively. The median total admission and ICU admission days were 15.5 and 9.0 days, respectively. Pneumonia and multiple organ

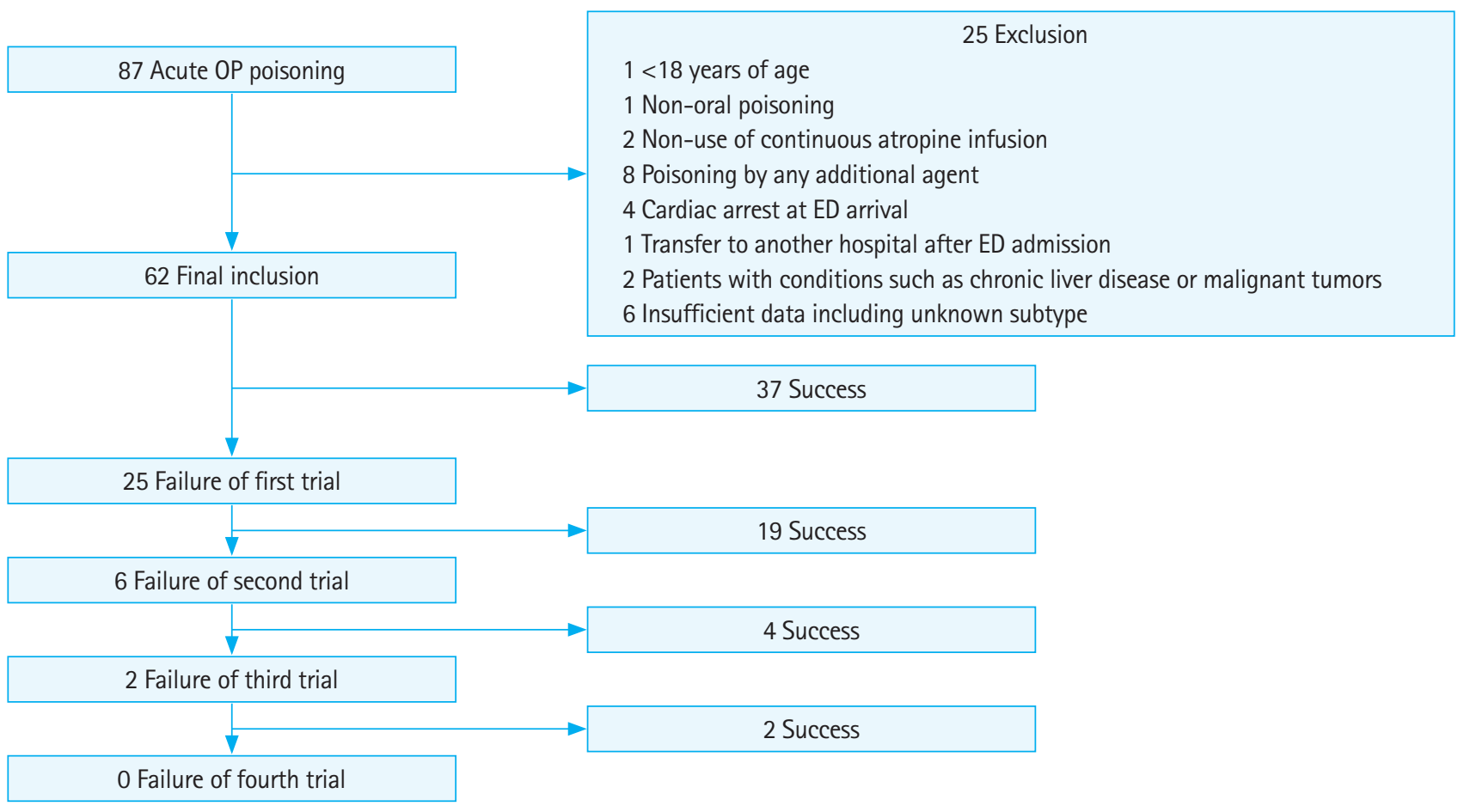

Fig. 1. Study flow chart. OP, organophosphate; ED, emergency department. 
Table 1. Baseline characteristics of patients with severe acute organophosphate poisoning

\begin{tabular}{|c|c|}
\hline Variable & Value $(n=62)$ \\
\hline Age (yr) & $60 \pm 16$ \\
\hline Sex, male & $47(75.8)$ \\
\hline \multicolumn{2}{|l|}{ Subtype of organophosphate } \\
\hline Dimethyl & $30(48.4)$ \\
\hline Diethyl & $16(25.8)$ \\
\hline Unclassified & $16(25.8)$ \\
\hline \multicolumn{2}{|l|}{ Namba classification } \\
\hline Moderate & 8 (12.9) \\
\hline Severe & $54(87.1)$ \\
\hline Ingested amount (mL) & $100(50-275)$ \\
\hline ED arrival time (hr) & $2.6(1.0-4.0)$ \\
\hline Charcoal & $51(82.3)$ \\
\hline Gastric lavage & $32(51.6)$ \\
\hline Intentionality & $52(83.9)$ \\
\hline \multicolumn{2}{|l|}{ Co-morbidity } \\
\hline Diabetes mellitus & $11(17.7)$ \\
\hline Hypertension & $18(29)$ \\
\hline Lung disease & $3(4.8)$ \\
\hline \multicolumn{2}{|l|}{ Vital signs } \\
\hline Systolic blood pressure (mmHg) & $136(108-161)$ \\
\hline Diastolic blood pressure $(\mathrm{mmHg})$ & 79 (63-89) \\
\hline Pulse rate (rates/min) & $98(84-112)$ \\
\hline Respiratory rate (rates/min) & $20(18-22)$ \\
\hline Body temperature $\left({ }^{\circ} \mathrm{C}\right)$ & $35.9(34.8-36.4)$ \\
\hline Initial Glasgow Coma Scale & $8(5-13)$ \\
\hline \multicolumn{2}{|l|}{ ST \& T change in ECG } \\
\hline ST elevation & $3(4.8)$ \\
\hline ST depression & $5(8.1)$ \\
\hline Initial PChE (U/L) & $322(300-1,386)$ \\
\hline \multicolumn{2}{|l|}{ Initial arterial blood gas } \\
\hline $\mathrm{pH}$ & $7.325(7.215-7.406)$ \\
\hline $\mathrm{PaO}_{2}(\mathrm{mmHg})$ & $96.3(73.5-126.3)$ \\
\hline $\mathrm{PaCO}_{2}(\mathrm{mmHg})$ & $35.2(28.9-43.6)$ \\
\hline Base excess (mmol/L) & $-1.9(-4.8--0.2)$ \\
\hline Lactate (mmol/L) & $4.380(2.598-7.553)$ \\
\hline \multicolumn{2}{|c|}{ No. of times until successful atropine discontinuation } \\
\hline First trial & $37(59.7)$ \\
\hline Second trial & 19 (30.6) \\
\hline Third trial & $4(6.5)$ \\
\hline Fourth trial & $2(3.2)$ \\
\hline Pneumonia & $23(37.1)$ \\
\hline Endotracheal intubation & $53(85.5)$ \\
\hline Respiratory failure & $52(83.9)$ \\
\hline Decreased mental status & $50(80.6)$ \\
\hline Acute kidney injury & $19(30.6)$ \\
\hline Hypotension & 19 (30.6) \\
\hline Total admission days & $15.5(8.0-29.5)$ \\
\hline ICU admission days & $9.0(5.0-18.3)$ \\
\hline In-hospital mortality & $8(12.9)$ \\
\hline
\end{tabular}

Values are presented as mean \pm standard deviation, number (\%), or median (interquartile range). Lung disease included asthma and chronic obstructive pulmonary disease.

$E D$, emergency department; ECG, electrocardiogram; PChE, plasma cholinesterase; ICU, intensive care unit. failure led to in-hospital mortality in 8 patients (12.9\%).

\section{Comparisons of PChE and serum lactate levels according to success or failure of atropine infusion discontinuation} Thirty-seven patients (59.7\%) experienced success on the first attempt to discontinue IV continuous atropine (Fig. 1). Comparisons of laboratory findings according to success or failure are shown in Table 2. There were no significant differences in the median interval from blood sampling to discontinuing IV atropine between successful and failed trials for either serum lactate sampling (5 vs. 5 hours, $\mathrm{P}=0.201$ ) or $\mathrm{PChE}$ sampling ( 5 vs. 4.5 hours, $\mathrm{P}=0.861$ ). Although PChE levels before trial discontinuation were higher in successful trial patients than in failed trial patients, there was no significant difference between groups $(1,713$ vs. $504 \mathrm{U} / \mathrm{L}, \mathrm{P}=0.075)$. Subgroup analysis revealed that in the dimethyl OP subtype, successful trial patients had significantly higher levels of PChE before the trial than failed trial patients $(2,439$ vs. $490 \mathrm{U} / \mathrm{L}, \mathrm{P}=0.030)$. There was also no significant difference between PChE level before trial discontinuation and initial PChE level (557 vs. $53 \mathrm{U} / \mathrm{L}$, $P=0.305)$. Subgroup analysis did not reveal any significant differences between $\mathrm{PChE}$ level before trial discontinuation and initial PChE level for any OP subtype. Serum lactate levels before trial discontinuation differed significantly between successful and failed trial groups (1.09 vs. $1.46 \mathrm{mmol} / \mathrm{L}, \mathrm{P}<0.001$ ). In subgroup analysis, patients with successful trials had significantly lower serum lactate levels before trial discontinuation regardless of OP subtype (dimethyl subtype: 1.08 vs. $1.41 \mathrm{mmol} / \mathrm{L}, \mathrm{P}=0.032$; diethyl subtype: 1.065 vs. $2.46 \mathrm{mmol} / \mathrm{L}, P=0.009$ ). Significantly more vasoactive drugs were used during trial atropine discontinuation in failed trials than in successful trials ( $3.2 \%$ vs. $15.2 \%, P=0.047$ ).

\section{Predictors of successful discontinuation of atropine in acute OP poisoning}

Serum lactate levels before trial discontinuation and use of vasoactive drugs were analyzed using multiple logistic regression to identify predictors related to successful discontinuation of atropine infusion in severe acute OP poisoning. Serum lactate (odds ratio, $0.516 ; 95 \% \mathrm{Cl}, 0.333$ to $0.801, \mathrm{P}=0.003$ ) was found to be the only significant predictor for all OP cases (Table 3 ). The AUC for prediction of successful atropine discontinuation using serum lactate levels before trial discontinuation was $0.742(95 \% \mathrm{Cl}, 0.638$ to 0.846 ) (Fig. 2). The cutoff value for prediction of successful atropine discontinuation using serum lactate levels before trial discontinuation was $1.13 \mathrm{mmol} / \mathrm{L}$ (sensitivity, 84.9\%; specificity, 58.1\%; positive predictive value, $51.9 \%$; negative predictive value, $87.8 \%$ ). Subgroup analysis demonstrated that the serum lactate level before trial discontinuation was a significant predictor regardless of 
Table 2. Comparison of laboratory findings according to success or failure in the discontinuation trial of continuous intravenous atropine

\begin{tabular}{|c|c|c|c|}
\hline Variable & Successful trial & Failed trial & P-value \\
\hline Total (95 attempts) & $n=62$ & $n=33$ & \\
\hline Use of vasoactive drug & $2(3.2)$ & $5(15.2)$ & 0.047 \\
\hline Presence of pneumonia & $22(35.5)$ & $14(42.4)$ & 0.507 \\
\hline PChE before trial (U/L) & $1,713(553-3,233)$ & $504(300-2,727)$ & 0.075 \\
\hline Difference in PChE before trial to initial PChE (U/L) & $557(0-1,888)$ & $53(0-1,283)$ & 0.305 \\
\hline $\mathrm{pH}$ & $7.421(7.377-7.472)$ & $7.376(7.323-7.463)$ & 0.077 \\
\hline $\mathrm{PaO}_{2}(\mathrm{mmHg})$ & $110.4(93.9-135.3)$ & $124.3(99.4-161.3)$ & 0.140 \\
\hline $\mathrm{PaCO}_{2}(\mathrm{mmHg})$ & $36.8(30.9-40.6)$ & $36.4(32.7-44)$ & 0.425 \\
\hline Base excess (mmol/L) & $-1.8(-3.88-1.05)$ & $-3.1(-5.65--0.35)$ & 0.171 \\
\hline Lactate $(\mathrm{mmol} / \mathrm{L})$ & $1.09(0.83-1.39)$ & $1.46(1.22-3.48)$ & $<0.001$ \\
\hline $\mathrm{pH}$ & $7.414(7.353-7.451)$ & $7.376(7.326-7.463)$ & 0.465 \\
\hline $\mathrm{PaO}_{2}(\mathrm{mmHg})$ & $108.2(97.8-140.3)$ & $125.7(103-144.7)$ & 0.413 \\
\hline $\mathrm{PaCO}_{2}(\mathrm{mmHg})$ & $37(33.2-41.2)$ & $34.3(30.8-43.5)$ & 0.886 \\
\hline Base excess (mmol/L) & $-1.85(-4.25-2.53)$ & $-2.4(-4.8--0.35)$ & 0.303 \\
\hline Lactate $(\mathrm{mmol} / \mathrm{L})$ & $1.08(0.83-1.72)$ & $1.41(1.13-3.48)$ & 0.032 \\
\hline Diethyl subtype (24 attempts) & $n=16$ & $n=8$ & \\
\hline PChE before trial $(\mathrm{U} / \mathrm{L})$ & $852(300-2,389)$ & $568(300-1,303)$ & 0.610 \\
\hline Difference in PChE before trial to initial PChE (U/L) & $0(-332-1,134)$ & $268(0-937)$ & 0.787 \\
\hline \multicolumn{4}{|l|}{ Arterial blood gas } \\
\hline $\mathrm{pH}$ & $7.436(7.386-7.488)$ & $7.36(7.251-7.467)$ & 0.238 \\
\hline
\end{tabular}

Values are presented as number (\%) or median (interquartile range).

PChE, plasma cholinesterase.

Table 3. Predictors of successful discontinuation of continuous atropine infusion in patients with severe acute organophosphate poisoning as determined by multivariate logistic regression analysis

\begin{tabular}{lcc}
\hline Variable & OR (95\% Cl) & P-value \\
\hline Total & & \\
$\quad$ Use of vasoactive drug & $0.230(0.038-1.411)$ & 0.112 \\
$\quad$ Serum lactate before trial (mmol/L) & $0.516(0.333-0.801)$ & 0.003 \\
Dimethyl subtype & $1.000(1.000-1.001)$ & 0.050 \\
$\quad$ PChE before trial (U/L) & $0.523(0.276-0.993)$ & 0.047 \\
$\quad$ Serum lactate before trial (mmol/L) & & \\
$\quad$ Diethyl subtype & $0.484(0.237-0.988)$ & 0.046 \\
$\quad$ Serum lactate before trial $(\mathrm{mmol} / \mathrm{L})$ & &
\end{tabular}

$\mathrm{OR}$, odds ratio; $\mathrm{Cl}$, confidence interval; $\mathrm{PChE}$, plasma cholinesterase.

\section{OP subtype (Table 3).}

In the dimethyl and diethyl subtype, the AUC for prediction of successful atropine discontinuation using the serum lactate level before trial discontinuation was $0.690(95 \% \mathrm{Cl}, 0.527$ to 0.854$)$ and $0.824(95 \% \mathrm{Cl}, 0.658$ to 0.991$)$, respectively.

\section{DISCUSSION}

Predicting successful atropine discontinuation helps physicians avoid unnecessary atropine toxicity. In this study, serum lactate levels before trial discontinuation were significantly lower in successful trials, and serum lactate was a significant predictor of successful atropine discontinuation for all OP cases and for both dimethyl and diethyl OP subtypes. Elevation of serum lactate levels generally reflects impaired tissue perfusion and is due to increased adenosine triphosphate production via anaerobic metabolism and decreased clearance by the liver, kidneys, and skeletal muscle. ${ }^{17,18}$ Increased serum lactate may develop due to OP-induced impaired ventilation and/or circulation. In this study, when 


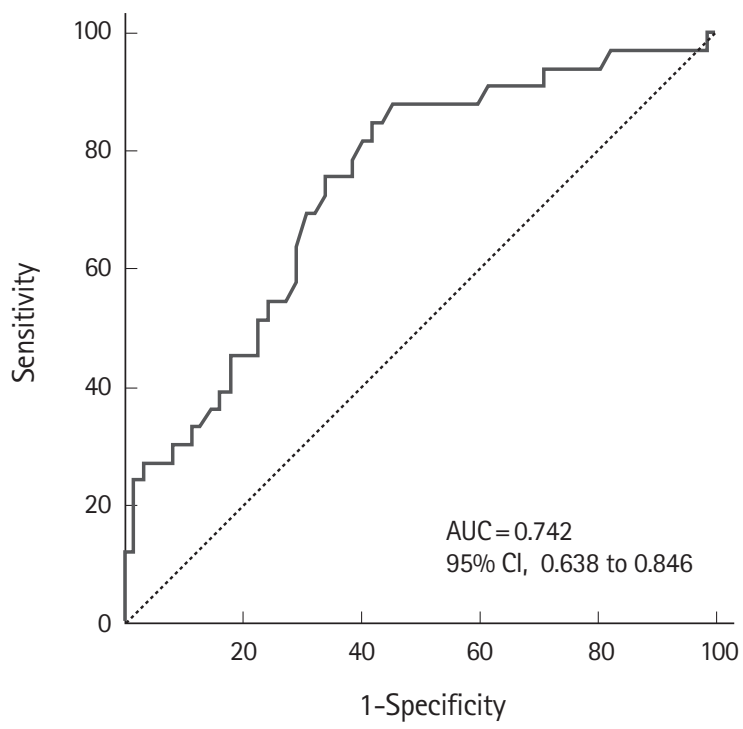

Fig. 2. Receiver operating characteristic curves of serum lactate before the trial in severe acute organophosphate poisoning. AUC, area under the receiver operating characteristics curve; $\mathrm{Cl}$, confidence interval.

trying to discontinue atropine, more vasoactive drugs such as norepinephrine were used to overcome shock in failed trials than in successful trials ( $15.2 \%$ vs. $3.2 \%, P=0.047)$. This suggests that even though blood pressure was within the normal range after use of vasoactive drugs, tissue perfusion was not as high as the tissue requirements owing to $\mathrm{OP}$-induced impaired circulation. Even though the toxic effects of OP were systemic, the cholinergic toxic effects of OP such as bronchorrhea and bronchospasm were not noticeable because they were masked by continuous atropine infusion; when atropine administration was discontinued, the cholinergic effects could still be observed. It is possible that discontinuation of atropine infusion fails when tissue perfusion is poor. Whether OP itself increases lactate levels has yet to be investigated; elevated lactate levels are also found in metformin and toxic alcohol poisoning. ${ }^{17}$ Therefore, whether the rise in lactate is a direct effect of OP and, if so, what level of serum lactate increase results from this direct effect will need to be investigated in future studies.

Although PChE levels before trial discontinuation trended to be higher in successful trials than in failed trials, the differences were not significant. There is wide physiological variation of PChE activity based on age, sex, and plasma lipid levels. ${ }^{19}$ Furthermore, PChE activity may change over the disease course owing to factors such as chronic liver disease and drug administration leading to inhibition of PChE activity or a reduction in its synthesis. ${ }^{19} \mathrm{~A}$ number of genetic mutants exist that result in PChE levels ranging from not measurable to higher than normal and daily variation in PChE enzyme activity in healthy individuals may be as high as $20 \%{ }^{19-21}$ Comparison of the absolute value of the PChE level before trial discontinuation may not be useful owing to these disadvantages. Therefore, we investigated changes in PChE levels before trial discontinuation as compared with initial PChE levels in the ED and found no significant differences. Based on the results of this study, the fact that PChE is easier to measure does not justify its use as an indicator of successful discontinuation of atropine infusion.

This study had some limitations. First, it was limited by its retrospective design. Missing data during collection was also a limitation. In particular, the ingested amount of toxin could have been overestimated or underestimated. Second, PChE inhibition varies between OPs. For example, most patients who die from chlorpyrifos poisoning have very low PChE activity when they present to the ED; however, since chlorpyrifos is a very potent PChE inhibitor, many other chlorpyrifos-poisoned patients who do not die also present with very low PChE activity. ${ }^{22}$ As this study was conducted at a single hospital and the sample size was small, we could not perform more detailed subgroup analyses. Third, since the exact percentages of OP, solvent, and surfactant were unknown, their clinical influence could not be predicted. Therefore, we could not assess the toxic effects of each component. However, because we usually do not see $\mathrm{OP}$ insecticide poisoning cases that contain only $\mathrm{OP}$ and because most $\mathrm{OP}$ insecticide products contain $\mathrm{OP}$ and similar solvents and surfactants, this study does have clinical utility. Fourth, we could not investigate genetic mutations resulting in adjusted PChE levels and R-AChE, which could be a more appropriate indicator of the severity of OP poisoning than $\mathrm{PChE}_{1}{ }^{20}$ because our facility could not test it.

In summary, the serum lactate level before a discontinuation trial of atropine infusion served as a predictor of successful trials in severe acute $\mathrm{OP}$ poisoning.

\section{CONFLICT OF INTEREST}

No potential conflict of interest relevant to this article was reported.

\section{REFERENCES}

1. Roth A, Zellinger I, Arad M, Atsmon J. Organophosphates and the heart. Chest 1993;103:576-82.

2. Sidell FR. Clinical effects of organophosphorus cholinesterase inhibitors. J Appl Toxicol 1994;14:111-3.

3. Eddleston M, Dawson A, Karalliedde L, et al. Early management after self-poisoning with an organophosphorus or carbamate pesticide: a treatment protocol for junior doctors. Crit 
Care 2004;8:R391-7.

4. Roberts DM, Aaron CK. Management of acute organophosphorus pesticide poisoning. BMJ 2007;334:629-34.

5. Eddleston $M$, Roberts $D$, Buckley N. Management of severe organophosphorus pesticide poisoning. Crit Care 2002;6:259.

6. del Portal DA, Shofer F, Mikkelsen ME, et al. Emergency department lactate is associated with mortality in older adults admitted with and without infections. Acad Emerg Med 2010; 17:260-8

7. Shapiro NI, Howell MD, Talmor D, et al. Serum lactate as a predictor of mortality in emergency department patients with infection. Ann Emerg Med 2005;45:524-8.

8. Ismail F, Mackay WG, Kerry A, Staines H, Rooney KD. The accuracy and timeliness of a Point Of Care lactate measurement in patients with Sepsis. Scand J Trauma Resusc Emerg Med 2015;23:68.

9. Karon BS, Scott R, Burritt MF, Santrach PJ. Comparison of lactate values between point-of-care and central laboratory analyzers. Am J Clin Pathol 2007;128:168-71.

10. Johnson MK. Mechanisms of and biomarkers for acute and delayed neuropathic effects of organophosphorus esters. In: Travis CC, editor. Use of biomarkers in assessing health and environmental impacts of chemical pollutants. Boston, MA: Springer; 1993. p.169-82.

11. Namba T, Nolte CT, Jackrel J, Grob D. Poisoning due to organophosphate insecticides. Acute and chronic manifestations. Am J Med 1971;50:475-92.

12. Levey AS, Levin A, Kellum JA. Definition and classification of kidney diseases. Am J Kidney Dis 2013;61:686-8.

13. Moon JM, Chun BJ, Lee BK. Glasgow coma scale score in the prognosis of acute carbamate insecticide intoxication. Clin
Toxicol (Phila) 2012;50:832-7.

14. Eddleston $M$, Worek F, Eyer P, et al. Poisoning with the S-Alkyl organophosphorus insecticides profenofos and prothiofos. OJM 2009;102:785-92.

15. Peter JV, Jerobin J, Nair A, et al. Clinical profile and outcome of patients hospitalized with dimethyl and diethyl organophosphate poisoning. Clin Toxicol (Phila) 2010;48:916-23.

16. Moon J, Chun B. Utility of red blood cell acetylcholinesterase measurement in mechanically ventilated subjects after organophosphate poisoning. Respir Care 2014;59:1360-8.

17. Kraut JA, Madias NE. Lactic acidosis. N Engl J Med 2014;371: 2309-19.

18. Levraut J, Ciebiera JP, Chave S, et al. Mild hyperlactatemia in stable septic patients is due to impaired lactate clearance rather than overproduction. Am J Respir Crit Care Med 1998;157 (4 Pt 1):1021-6.

19. Ostergaard D, Jensen FS, Viby-Mogensen J. Pseudocholinesterase deficiency and anticholinesterase toxicity. In: Ballantyne B, Marrs TC, editors. Clinical and experimental toxicology of organophosphates and carbamates. Stoneham, MA: Butterworth-heinemann; 1992. p.520-7.

20. Karalliedde LD, Edwards P, Marrs TC. Variables influencing the toxic response to organophosphates in humans. Food Chem Toxicol 2003;41:1-13.

21. Gallo MA, Lawryk NJ. Organic phosphorus pesticides. In: Hayes WA, Laws ER, editors. Handbook of pesticide toxicology. New York, NY: Academic Press; 1991. p.917-1123.

22. Eddleston $M$, Eyer $P$, Worek F, Sheriff MH, Buckley NA. Predicting outcome using butyrylcholinesterase activity in organophosphorus pesticide self-poisoning. OJM 2008;101:467-74. 